\title{
Controlling Unstable Rolling Phenomena
}

BILL GOODWINE

Department of Aerospace and Mechanical Engineering, Univerity of

Notre Dame, Notre Dame, Indiana 46556

GÁBOR STÉPÁN

Department of Applied Mechanics, Technical University of Budapest, H-1521 Budapest, Hungary

Abstract: The paper addresses dynamic and control issues related to a dynamical model called the classical shimmying wheel. The classical shimmying wheel models the rolling dynamics of many physical rolling systems such as aircraft nose wheels, motorcycles, automotive systems and tractortrailer systems. Such system can exhibit undesirable unstable rolling motion, i.e. shimmying, which can often lead to disastrous results.

Prior work with this particular model has focused on the stability of the system as well as an analysis of the qualitative nature of its dynamics, including numerical observation of possible chaotic behavior. Such behavior is observed when the rolling element is allowed to slip under certain conditions, which is intended to realistically model real physical rolling systems. In cases where the rolling dynamics of the system are unstable, the dynamics are characterized by the presenece of an attractor wherein the system repeatedly switches back and forth between rolling and slipping. We present a slightly different, but more realistic, condition for the rolling element to switch from pure rolling to a slipping state and observe similar behavior.

Additionally, we present a controller for the system designed using the method of feedback linearization. This controller stabilizes the purely rolling system but fails to always stabilize the system which is allowed to slip. We investigate the conditions under which the controller stabilizes the slipping system and propose an effective alternative control strategy for the slipping system for the case when the original controller fails to stabilize the system and where the uncontrolled rolling system is unstable. Finally, we investigate the stability of the system about operating points which are not equilibrium points, which models a physical system executing a turn.

Keywords: nonlinear control, nonholonomic systems, shimmy, hybrid systems. 


\section{INTRODUCTION}

Mechanical systems which contain rolling elements are naturally modeled as nonholonomic mechanical systems. However, the "rolling without slipping" assumption is clearly an approximation and is not valid, for example, for elastic rolling contact above a certain speed (see e.g., (Pacejka, 1988)). Additionally, for real physical rolling systems, the rolling without slipping constraint is imposed by friction. However, since a friction force has a limited magnitude, if the nonholonomic constraint force exceeds that limit, the real rolling system will transition from a rolling state to a skidding state. We will refer to this more realistic situation as the "skidding system," but we emphasize that such a system can also exhibit pure rolling behavior, i.e., the system may alternatively transition from rolling to skidding, and viceversa. It will skid only if the nonholonomic constraint force exceeds that which can be supplied by friction. We will refer to the system that can not skid as the purely rolling system or the nonholonomic system. Throughout this paper we will use the terms "slip" and "skid" interchangeably. In both cases, to slip or skid means that the relative velocity between the point of contact of the wheel and the surface on which it is rolling is not zero. We use the term "slip" because of its use in the common phrase "rolling without slipping" in the nonholonomic literature, and the term "skid" because of its common use in vehicle dynamics.

One particular model, we will refer to as the classical shimmying wheel, is the focus of this paper. Unstable rolling is obviously a very important phenomenon in vehicle dynamics, affecting many system from aircraft nose wheels, truck trailers and motorcycle front wheels to the ubiquitous shimmying shopping cart wheel. Although, for pneumatic tires in particular, more sophisticated models now exist, see, e.g., (Nimark and Fufaiv, 1972) and (Böhm and Kollatz, 1989), the simpler model we consider still describes the complicated dynamics of a shimmying system, and gives a tractable topological and geometric structure of the dynamics. In the case where the system is allowed to skid, (Stépán, 1991) observed numerical experimental evidence of chaotic behavior and presented an approximate one dimensional map explaining the chaotic and transient chaotic motions. As we will illustrate, certainly the complicated qualitative nature of the dynamics is not lost by restricting our attention to the simplified wheel model.

One conclusion of this paper is a negative answer to the following question: if a nonholonomic system is in some sense "stable," (possibly stabilized by a controller) is the same also true for the more realistic system which is allowed also to slip or skid? This question is particularly important in the 
context of control theory, since if it is true, then one can design a stabilizing controller for the nonholonomic system (which may be an easier task than for a skidding system involving kinetic Coulomb friction since it has a lower dimensional state space), and be assured that it also stabilizes the skidding system. In this paper we present an example of one system for which this is not true. We design a controller for the nonholonomic system and then demonstrate that it is not stabilizing for the more realistic model where the constraints are imposed by Coulomb friction.

Since the controller designed for the nonholonomic system does not always stabilize the skidding system, it is necessary to resort to alternative means to stabilize the skidding system. One possible means of stabilizing the system would be to utilize the existence of the attractor observed in (Stépán, 1991). A strategy which does this would be to allow the controller to attempt to stabilize the system only when it is rolling. When the system is skidding, the dissipative nature of the skidding will drive the system to the attractor, which is characterized by frequent switching between rolling and skidding, and vice-versa. We will demonstrate that this approach is very effective.

Finally, since the controller designed renders the pure rolling system fully controllable, we investigate the stability of the system about operating points which are not equilibrium points. Such situations are intended to model a real physical system, such as a motorcycle, executing a turn, rather than rolling along a straight line. We simulate the system transitioning between the equilibrium operating point (rolling straight ahead) and a nonequilibrium operating point (turning), and compare the performance of our controller with that of a linear controller.

In Section 2, we present the model under consideration, the equations of motion, and present and review the qualitative nature of the dynamics. In Section 3, we review the notion of feedback linearization and construct a controller for this system using that technique. In Section 4 we present simulation results for the system with the controller for both the nonholonomic system as well as the skidding system. Finally, in Section 5 we present simulations results for the turning system.

\section{THE DYNAMICS OF THE CLASSICAL SHIM- MYING WHEEL}

In this section, we describe the model and its dynamics. A schematic drawing of the model is shown in Figure 1. The rotational angle of the wheel 
with radius $r$ is given by $\phi$. The caster length, or the offset of the axis of the wheel with respect to the vertical center of rotation of the wheel (the kingpin) is $l$, and the angle of rotation of the wheel assembly with respect to the "straight" position is given by $\theta$. We will consider the kingpin to be massless. Call the mass of the connecting assembly $m_{c}$ and the mass of the wheel $m_{w}$. For the control problem, we will consider the control input to be a torque, $u$, about the vertical center of rotation of the wheel assembly.

In this study, the simplest possible mechanical model is considered, with the lowest number of degrees of freedom which still exhibits the shimmying instability. This goal of simplicity perhaps makes the model less similar to a particular example, e.g., less like an automobile suspension. On the other hand, reducing the problem to the simplest possible model serves a two-fold purpose. First, the problem becomes tractable, allowing the geometry of the dynamics to be explored. Second, by considering the simplest possible model, we hope to reduce the rather general phenomena, present in many different applications, to its essential elements.

The main simplification of this model is that the elastic nature of the system is modeled by springs; whereas, the more sophisticated models mentioned previously directly attempt to model the infinite dimensional elastic nature of pneumatic tires, or possibly reduce the problem to a finite dimensional representation by only considering the lower order modes (Sharp and Jones, 1980). However, there are some cases where our simplified model may be a more accurate model than the more complicated ones. For example, on an aircraft with a relatively tall landing gear structure, the elastic effect of the tire may be small relative to the lateral elastic properties of the landing gear strut. Another case is whenever there is a small contact region, or if the wheel is rigid (as in a shopping cart). Regardless, here we model the elastic element by two springs, each with spring constant $\frac{k}{2}$. The kingpin is constrained to deflect laterally (so it can not deflect "forwards and backwards", but only "side to side"), and the amount of deflection is represented by the variable $y$.

We consider the system to be moving with a constant velocity, $v$. This assumption further reduces the dimension of the phase space, thus helping to further simplify the problem. Such an assumption is justified in cases where the body is massive relative to the mass of the wheel and the associated structure (as in an airplane), or where some external control keeps the overall structure moving with constant velocity (such as a truck trailer or shopping cart). Finally, we note that many structures such as an aircraft landing gear system or automobile suspension also include significant vertical elastic elements, e.g., the shock absorbers. Clearly, a model including 
these elements would be more realistic and could possibly alter the dynamics of the system. However, since the simpler, planar model we consider exhibits the phenomenon we wish to control, we will restrict our attention to the simpler model for the reasons set forth above.

\subsection{Dynamics of the Pure Rolling System}

The equations of motion for this system with the ideal nonholonomic constraining forces and control input $u$ are given by:

$$
\begin{aligned}
\ddot{\theta} & =\frac{-\frac{v}{l}\left(\sec ^{2} \theta-\frac{1}{2}+\frac{3 m_{w}}{2 m_{c}} \tan ^{2} \theta\right) \dot{\theta}}{\left(\frac{1}{3}+\tan ^{2} \theta\right) \cos \theta+\frac{m_{w}}{4 m_{c}}\left(\frac{r^{2}}{l^{2}} \cos \theta+6 \tan ^{2} \theta \cos \theta\right)} \\
& -\frac{\frac{k}{l m_{c}} y+\left(1+\frac{3 m_{w}}{2 m_{c}}\right) \frac{\tan \theta}{\cos \theta} \dot{\theta}^{2}-\frac{\cos \theta}{l^{2} m_{c}} u}{\left(\frac{1}{3}+\tan ^{2} \theta\right) \cos \theta+\frac{m_{w}}{4 m_{c}}\left(\frac{r^{2}}{l^{2}} \cos \theta+6 \tan ^{2} \theta \cos \theta\right)} \\
\dot{y} & =l \dot{\theta} \cos \theta+(v+l \dot{\theta} \sin \theta) \tan \theta \\
\dot{\phi} & =\frac{\sec \theta(v+l \dot{\theta} \sin \theta)}{r} .
\end{aligned}
$$

A complete study of the dynamics of this system is presented by (Stépán, 1991). In particular, it was shown that the linearized stability of the system about the $\theta=0$ and $y=0$ position was governed by the following condition:

$$
\frac{l}{r}>\sqrt{\frac{3 m_{w}}{2 m_{c}}}
$$

If the above condition is satisfied, the equilibrium point is asymptotically stable, and the equilibrium point is unstable otherwise. Additionally, it was shown, that when the above local stability condition is satisfied, an unstable limit cycle exists around the stable stationary motion. This stability condition does not contain the velocity term, which may seem to contradict intuition because in vehicle dynamics, the notion of a "critical speed" is often utilized. However, in the case presented here, equation 2 does not contain a velocity term because we have not included viscous damping in the equations of motion.

If we set the control input to zero, we can numerically verify and observe the above results. For $m_{c}=1.5 \mathrm{~kg}, m_{w}=2.75 \mathrm{~kg}, l=0.2 \mathrm{~m}, r=0.1 \mathrm{~m}, k=$ $75 \mathrm{~N} / \mathrm{m}$ and $v=1 \mathrm{~m} / \mathrm{s}$, the value of the critical caster length is $l_{\mathrm{cr}} \approx 0.1658 \mathrm{~m}$, 
so the length of the caster is greater than the critical length, and so the equilibrium solution is stable. Figures 2 and 3 show this stabile equilibrium solution. For a decreased caster length, $l=0.152 \mathrm{~m}<l_{\mathrm{cr}}$, Figures 4 and 5 show the local instability of the equilibrium solution. This unstable solution appears to be growing unbounded in all variables except $\theta$, which is bounded by $\pm \frac{\pi}{2}$.

The previous simulations verify that with certain parameter values, the equilibrium point can be locally unstable. Next, we numerically verify that even if the equilibrium point is locally stable, there exists an unstable limit

cycle around it. Figure 6 shows two solutions, with initial conditions which are "close" together, one of which is stable, the other of which is unstable. The first solution has initial conditions leading inside the limit cycle, and the second solution has initial conditions leading outside the limit cycle. In this simulation, we use the same physical parameters for the system as for the simulation demonstrating the local stability of the equilibrium solution, except $l=0.171 \mathrm{~m}>l_{\mathrm{cr}}$, which still satisfies the local stability condition expressed by equation 2 . In both cases, the initial conditions are all zero, except for the solution leading inside the limit cycle, the initial angle is $\theta_{0}=-0.24$ and the initial angular velocity is $\dot{\theta}_{0}=0.4 \mathrm{~s}^{-1}$. For the solution leading outside the limit cycle, $\theta_{0}=-0.24$ and $\dot{\theta}_{0}=0 \mathrm{~s}^{-1}$. See the bifurcation analysis in (Stépán, 1991) for more details.

\subsection{Dynamics of the Skidding System}

In order to properly model a skidding system, we must first address the manner in which we model frictional effects. This is a difficult issue because a general theory for frictional phenomena does not exist. See, for example, (Bidwell, 1962), (Stanton, 1923), (Blau, 1996) and (Gemant, 1950). Although not explicitly recognized, for the rolling system in the previous section, we have made the simplifying assumption of choosing not to incorporate rolling resistance into the model. For the skidding system, we similarly simplify the complicated frictional effects. The previous references provide empirical evidence that the coefficient of friction for skidding systems is dependent upon the skidding velocity. Indeed, as the skidding velocity increases, the coefficient of friction decreases. In our case, however, we will choose to model the coefficient of skidding friction as a constant by adopting the standard Coulomb friction model.

We will assume that the dynamics of the system switch from a pure rolling system to a skidding system if the magnitude of the constraining 
force exceeds that which can be applied by friction, i.e.,

$$
\left\|f_{c}\right\|>\left(\frac{1}{2} m_{c}+m_{w}\right) g \mu_{s}
$$

where $\mu_{s}$ is the coefficient of static friction and $f_{c}$ is the nonholonomic constraint force.

We will assume that the dynamics of the system switch from skidding to pure rolling if the absolute value of the relative velocity of the point of contact of the wheel and the surface is less than some small, specified value. In all the simulation results presented, we take this value to be $0.02 \mathrm{~m} / \mathrm{s}$. This assumption furthers two purposes. First, since the coefficient of friction increases as relative velocity decreases, then modeling the switch to rolling when the relative velocity becomes "small" rather than zero may capture some of this effect in the model. Secondly, since the relative skidding velocity determines the direction in which the frictional force acts, as the relative velocity becomes very small, it will be numerically difficult to accurately resolve the direction in which the force acts. For a more complete exposition, see the treatment for the so-called "stick slip" phenomena in (Gemant, 1950).

We have given the equations of motion for the purely rolling system in equation 1 . The equations of motion for the skidding system are:

$$
\begin{gathered}
\left(\begin{array}{ccc}
\left(\frac{1}{3} m_{c}+\left(1+\frac{r^{2}}{4 l^{2}} m_{w}\right) l^{2}\right. & -\left(\frac{1}{2} m_{c}+m_{w}\right) l \cos \theta & 0 \\
-\left(\frac{1}{2} m_{c}+m_{w}\right) l \cos \theta & m_{c}+m_{w} & 0 \\
0 & 0 & \frac{1}{2} m_{w} r^{2}
\end{array}\right)\left(\begin{array}{l}
\ddot{\theta} \\
\ddot{y} \\
\ddot{\phi}
\end{array}\right)= \\
\left(\begin{array}{c}
\frac{v_{p_{x}}}{\sqrt{v_{p_{x}}^{2}+v_{p_{y}}^{2}}}\left(\frac{1}{2} m_{c}+m_{w}\right) l_{\mu} g+u \\
-\left(\frac{1}{2} m_{c}+m_{w}\right) l \dot{\theta}^{2} \sin \theta-k y-\frac{v_{p_{x}} \sin \theta+v_{p_{y}} \cos \theta}{\sqrt{v_{p_{x}}^{2}+v_{p_{y}}^{2}}}\left(\frac{1}{2} m_{c}+m_{w}\right) \mu_{d} g \\
\frac{v_{p_{x}}}{\sqrt{v_{p_{x}}^{2}+v_{p_{y}}^{2}}}\left(\frac{1}{2} m_{c}+m_{w}\right) r \mu_{d} g
\end{array}\right),
\end{gathered}
$$

where $v_{p_{x}}$ and $v_{p_{y}}$ represent the two components of the velocity of the point of contact of the wheel relative to the surface and are functions of the variables $\theta, \dot{\theta}, \dot{y}$ and $\dot{\phi}$, and $\mu_{d}$ is the coefficient of dynamic (skidding) friction.

Clearly, for this system, the phase space is six-dimensional, and the dynamics of the system when it is allowed to switch between rolling and skidding will switch back and forth between a four-dimensional phase space (the rolling, nonholonomic system) and the six-dimensional system given in 
equation 4. Given the strong nonlinearities present in the system, which are, in fact, discontinuous when the system switches between the four and sixdimensional dynamics, highly nonlinear and possibly even chaotic behavior is not unexpected.

The topology of the phase space of this system is fully discussed by (Stépán, 1991). Briefly, in the case of pure rolling, in the $(\theta, \dot{\theta}, y)$ phase plane, there is a strongly attractive stable two-dimensional center manifold. This center manifold is readily observed in the simulations presented in Figures 3 and 6 .

The rolling without slipping requirement, given by the nonholonomic constraint, defines a four-dimensional submanifold of the six-dimensional phase space upon which the system is purely rolling. The fact that the constraints define a submanifold of the phase space is easily verified using the preimage theorem. In this four-dimensional submanifold, equation 3 determines the regions where the constraint forces exceed in magnitude that which can be supplied by friction. For the system to switch from skidding to rolling, the solution must intersect the four-dimensional "rolling" submanifold of the six-dimensional phase space in a region where the nonholonomic constraint for is less than the maximum friction force. The system will then evolve on the four-dimensional "rolling" submanifold until the solution enters a "skidding" region defined by equation 3 .

In the following simulations, $f_{c}$ in equation 3 is evaluated using the Lagrange multipliers which were the by-product of the original derivation of the equations of motion and is a function of the physical parameters and the coordinates $(\theta, \dot{\theta}, y)$. This may lead to results which are slightly different than in (Stépán, 1991), which used an approximation of equation 3. If we denote the Lagrange multipliers by $\lambda_{1}$ and $\lambda_{2}$, then the level set

$$
f_{c}=\sqrt{\lambda_{1}^{2}+\lambda_{2}^{2}}=\left(\frac{1}{2} m_{c}+m_{w}\right) g \mu_{s}
$$

describes the set of points in the phase space where the nonholonomic constraint force equals the maximum friction force. Figure 7 shows the top and bottom "halves" of this level set for the physical parameter values $m_{c}=1.5 \mathrm{~kg}, m_{w}=2.75 \mathrm{~kg}, l=0.2 \mathrm{~m}, r=0.1 \mathrm{~m}, k=75 \mathrm{~N} / \mathrm{m}, v=1 \mathrm{~m} / \mathrm{s}$ and $\mu_{s}=0.05$.

We note that typically in of our simulations, $|y|<0.01$, so the level surface is approximated well by its geometry at $y=0$. This level curve is illustrated in Figure 8. In (Stépán, 1991), this level curve was approximated by two planes. It is evident from the figure and the similarity of our results with (Stépán, 1991) approximation is valid for the cases we are considering 
in this paper. A more global investigation, including possible ways to exploit the geometry of the system illustrated in Figure 7 for control purposes will be the subject of a future publication.

A simulation with the same parameter values as in the first simulation where the equilibrium point is locally stable, and with a coefficient of static (pure rolling) friction, $\mu_{s}=0.18$ and a coefficient of dynamic (sliding or skidding) friction of $\mu_{d}=0.09$ is shown in Figure 9. Figure 10 shows an enlarged portion of the trajectory. In this simulation, all the physical parameters are the same as in the previous simulations, except $l=0.171 \mathrm{~m}>l_{c r}$. However, the initial conditions for this simulation were taken to be outside the unstable limit cycle.

We note that if we carefully observe Figure 10, there appears to be a complicated periodic orbit involving multiple switches between the rolling and skidding dynamics. As mentioned previously, prior investigation in (Stépán, 1991) suggests that this system is chaotic. Our investigation suggests the possibility that chaotic motion may result from repeated "bifurcations" in the number of switches between rolling and skidding. While this issue is certainly intriguing, we defer a full investigation of a complete investigation of the dynamics since the primary focus of this paper is controlling this complicated dynamic phenomenon. What we emphasize, however, is the existence of attractive dynamics. In (Stépán, 1991), numerical evidence of a chaotic attractor was presented. Similarly, here we observe that the dynamics of the system are attracted to an alternative rolling and slipping regime. The reason for this is intuitively obvious: the skidding dynamics are dissipative, which is inherent in the Coulomb friction model, which naturally drives the system to the rolling dynamics. Alternatively, unstable rolling dynamics will cause the system to eventually switch to skidding since the constraint forces will eventually exceed the Coulomb static friction limit. This will result in the system alternatively switching back and forth between rolling and skidding, as illustrated in Figures 9 and 10.

\section{FEEDBACK LINEARIZATION OF THE CLAS- SIC SHIMMYING WHEEL MODEL}

In this section we review the subject of control via feedback linearization and apply the technique to the shimmying wheel model. 


\subsection{Review of Feedback Linearization}

Here we briefly summarize the concept of feedback linearization. A complete description can be found in (Isidori, 1989), (Nijmeijer and der Schaft, 1990) and (Hauser et al., 1992) and related work in differential flatness can be found in (Fliess et al., 1992) and (van Nieuwstadt et al., 1998). Although these two concepts are related, and in some cases essentially equivalent, they are in some sense philosophically different. The basic idea in feedback linearization is to find a (generally non-linear) change of coordinates which transforms the system into one which is linear and controllable. On the other hand, in flatness the focus is more on generating feasible trajectories. For a complete comparison, see (van Nieuwstadt and Murray, 1996). Since we are primarily concerned with stabilizing the the shimmying, we adopt the feedback linearization framework.

Consider the single input, $u$, single output, $y$, control system:

$$
\Sigma_{0}=\left\{\begin{array}{l}
\dot{x}=f(x)+g(x) u \\
y=h(x)
\end{array}\right.
$$

where $h$ is called the output function, $u$ is the control input and $\operatorname{dim}(x)=n$. This system is said to have relative degree $r$ at a point $x_{0}$ if

1. $L_{g} L_{f}^{k} h(x)=0$ for all $x$ in a neighborhood of $x_{0}$ for $k=0,1, \ldots,(r-2)$, and

2. $L_{g} L_{f}^{r-1} h\left(x_{0}\right) \neq 0$,

where $L_{g} h$ is the Lie derivative of the function $h$ with respect to the vector field $g$. In coordinates,

$$
L_{f} h(x)=\sum_{i=1}^{n} \frac{\partial h}{\partial x_{i}} f_{i}(x) .
$$

If the relative degree of the control system is equal to its dimension, i.e., $r=n$, at all points $x$, then the system is said to be full state feedback linearizable, and we can employ the following change of coordinates:

$$
\begin{aligned}
\xi_{1}= & h \\
\xi_{2}= & \dot{\xi}_{1}=L_{f} h(x) \\
\xi_{3}= & \dot{\xi}_{2}=L_{f}^{2} h(x) \\
& \vdots \\
\xi_{n}= & \dot{\xi}_{n-1}=L_{f}^{n-1} h(x) \\
& \dot{\xi}_{n}=L_{f}^{n} h(x)+L_{g} L_{f}^{n-1} h(x) u .
\end{aligned}
$$


Since $L_{g} L_{f}^{n-1} h \neq 0 \forall x$, we can define

$$
u=\frac{1}{L_{g} L_{f}^{n-1} h}\left(-L_{f}^{n} h+\nu\right)
$$

so that $\dot{\xi_{n}}=\nu$.

In this manner, the nonlinear control system $\Sigma_{0}$ has been transformed into a linear system (a chain of integrators), which is in controllable canonical form. Then, we simply use standard linear theory to design the transformed control, $\nu$.

Since this system is linear and controllable, it is also stabilizable. We can also specify an arbitrary trajectory by specifying the value of $h$ along it. If $h_{d}(x(t))$ is the desired "trajectory," we can choose

$$
\nu=h_{d}^{(n)}+\alpha_{n-1}\left(h_{d}^{(n-1)}-\xi_{n}\right)+\cdots+\alpha_{0}\left(h_{d}-\xi_{1}\right),
$$

where the $\alpha_{i}$ are chosen so that $s^{n}+\alpha_{n-1} s^{n-1}+\cdots+\alpha_{1} s+\alpha_{0}$ is a stable Hurwitz polynomial. Arbitrary trajectories may or may not be possible, depending upon the form of the output function, $h(x)$ because the feasible trajectories must be compatible with that function.

We note that there are alternative formulations to the feedback linearization problem and refer the reader to (Nijmeijer and der Schaft, 1990) for more details. Clearly, the most difficult aspect of the problem as we have presented it is determining the output function $h(x)$ which satisfies the requirements of the partial differential equations appearing in the definition of relative degree.

\subsection{Full State Feedback Linearization of the Shimmying Wheel}

The main difficulty in applying the feedback linearization method to a given problem is determining the output function. In the case of the classical shimmying wheel (the purely rolling case), we have

$$
\begin{gathered}
x=(\theta, \dot{\theta}, y)^{T} \\
f(x)+g(x) u=(\dot{\theta}, \ddot{\theta}, \dot{y})^{T}
\end{gathered}
$$

where $\dot{y}, \dot{\phi}$ and $\ddot{\theta}$ are given by equation 1 . The control input vector field, $g(x)$ is simply the terms in $\ddot{\theta}$ which contain the control input term, $u$ and the drift vector field $f(x)$ is the remaining terms. Both of these vector fields can easily be identified from equation 1 : 


$$
f(x)=\left(\begin{array}{c}
\dot{\theta} \\
\frac{-\frac{v}{l}\left(\sec ^{2} \theta-\frac{1}{2}+\frac{3 m_{w}}{2 m_{c}} \tan ^{2} \theta\right) \dot{\theta}-\frac{k}{2 m_{c}} y+\left(1+\frac{3 m_{w}}{2 m_{c}}\right) \frac{\tan \theta}{\cos \theta} \dot{\theta}^{2}}{\left(\frac{1}{3}+\tan ^{2} \theta\right) \cos \theta+\frac{m_{w}}{4 m_{c}}\left(\frac{r^{2}}{l^{2}} \cos \theta+6 \tan ^{2} \theta \cos \theta\right)} \\
l \dot{\theta} \cos \theta+(v+l \dot{\theta} \sin \theta) \tan \theta
\end{array}\right)
$$

and

$$
g(x)=\left(\begin{array}{c}
0 \\
\frac{-\frac{\cos \theta}{l^{2} m_{c}}}{\left(\frac{1}{3}+\tan ^{2} \theta\right) \cos \theta+\frac{m_{w}}{4 m_{c}}\left(\frac{r^{2}}{l^{2}} \cos \theta+6 \tan ^{2} \theta \cos \theta\right)} \\
0
\end{array}\right) .
$$

Rather than attempt to solve the partial differential equations defined in the definition of relative degree, in this case, we can adopt a more ad hoc approach. In order to determine an output function, note that if we take $h_{1}(x)=y$,

$$
\begin{aligned}
L_{g} h_{1} & =0 \\
L_{f} h_{1} & =l \dot{\theta} \cos \theta+(v+l \dot{\theta} \sin \theta) \tan \theta \\
L_{g} L_{f} h_{1} & \neq 0 .
\end{aligned}
$$

Since $L_{g} L_{f} h_{1} \neq 0, h_{1}$ is not an output function which renders the system feedback linearizable. Note, however, that if another output function, $h_{2}(x)$ were purely a function of $\theta$, we would have

$$
L_{f} h_{2}=\frac{d h_{2}}{d \theta} \dot{\theta}
$$

since the $\theta$ component of $f$ is simply $\dot{\theta}$. Since $L_{f} h_{1}$ is linear in $\dot{\theta}$, and otherwise only a function of $\theta$, we can differentiate it with respect to $\dot{\theta}$, integrate it with respect to $\theta$, and subtract the result from $h_{1}$. If we denote the resulting function by $h$, we have

$$
h(\theta, \dot{\theta}, y)=-y+\log \frac{\cos \left(\frac{\theta}{2}\right)+\sin \left(\frac{\theta}{2}\right)}{\cos \left(\frac{\theta}{2}\right)-\sin \left(\frac{\theta}{2}\right)}
$$

In this manner, we have effectively cancelled all the terms in $L_{f} h$ which would lead to non-zero terms in $L_{g} L_{f} h$, because the only non-zero component of $g$ is the $\dot{\theta}$ component, and we have cancelled the $\dot{\theta}$ functionality of $L_{f} h$ by our construction. 
Also note that

$L_{g} L_{f}^{2} h=\frac{24 v}{l^{2}\left(16 m_{c}+18 m_{w}\right)+3 m_{w} r^{2}+\left(-2 l^{2}\left(4 m_{c}+9 m_{w}\right)+3 m_{w} r^{2}\right) \cos (2 \theta)}$

which is clearly globally non-zero for non-zero $v$. Whether such a construction is possible for general nonholonimc systems is certainly an intriguing question, but one which we will defer until a later investigation.

\section{CONTROLLER SIMULATIONS}

In this section, we numerically implement the controller designed in Section 3 on both the purely rolling system as well as the system which is allowed to slip or skid.

\subsection{The Purely Rolling System}

Here we implement the controller on the purely rolling system. In the first simulation we take the physical parameters to be the same as for the simulation shown in Figure 6 and the initial conditions to be "outside" the unstable limit cycle, so that without the controller the system would be unstable. These are the same initial conditions which lead to the unstable solution illustrated in Figure 6. Figure 11 illustrate the expected asymptotic stability.

Next, we verify that the controller stabilizes the system when the physical parameters do not satisfy the stability criterion for local stability of the equilibrium solution. In this case, we take $l=0.152<l_{c r}$ and the initial conditions $(\theta, \dot{\theta}, y)=(-0.75,0,0)$. The result appears in Figure 12 .

\subsection{The Skidding System}

In this section, we attempt to use our controller to control the more realistic system which will skid if the nonholonomic constraint force exceeds the maximum friction force. Recall that in the uncontrolled case the dynamics of this system were characterized by (repeated) switches between rolling and skidding.

\subsubsection{The Obvious Approach}

The obvious first attempt would be simply to use the same controller on the skidding system, ignoring the fact that the skidding system is actually 
different. Figures 13 show the results from when we test the controller on the system when it is allowed to skid. In this simulation the physical parameters are $m_{c}=1.5 \mathrm{~kg}, m_{w}=5.75 \mathrm{~kg}, l=0.2 \mathrm{~m}, r=0.1 \mathrm{~m} k=75.0 \mathrm{~kg} / \mathrm{m}$ (which makes the equilibrium solution locally unstable) and $\mu_{s}=0.4$ and $\mu_{d}=0.2$. We take the initial conditions to be "way out" into the skidding regime:

$$
(\theta, y, \phi, \dot{\theta}, \dot{y}, \dot{\phi})=(-0.5,0,0,0,0,0) \text {. }
$$

Note, however, for the same initial conditions, but for a smaller coefficient of friction, $\mu_{s}=0.1$, and $\mu_{d}=0.2$, the controller fails to stabilize the system. See Figure 14 . Note that as $\theta$ approaches $\frac{\pi}{2}$, the controller becomes unbounded.

These, and other, numerical experiments indicate that the controller is stabilizing for a region of the phase space that gets larger as the coefficient of friction increases. The intuitive reason for this is obvious: the larger the coefficient of friction is, the "closer" the system is to that for which the controller is actually designed.

\subsubsection{An Alternative Approach}

If we adopt a slightly different approach, however, better results are obtained. If the initial conditions for the system are in the slipping regime and the parameter values for the system are such that an attractor exists, the solution will approach the attractor. If we set the control input to zero until the solution is near this attractor, indicated by a switch from slipping to rolling, better results are obtained. Figure 15 shows the solution for a system with the same physical parameters and same initial conditions as the preceding (unstable) simulation.

At least with regard to numerical experimentation, this control strategy seems globally (with respect to initial conditions in $\theta$ ) stabilizing. Figures 16 and 17 show the results when the initial conditions are

$$
(\theta, y, \phi, \dot{\theta}, \dot{y}, \dot{\phi})=(3.0,0,0,0,0,0) .
$$

which is virtually completely backwards. Indeed, in over 5000 numerical experiments, this control strategy stabilized the system every time, except in the case where $\mu_{s}=\mu_{d}$, which, fortunately, is a physically unrealistic situation.

\subsection{Comparison with Simple Linear Controller}

Here we briefly compare the performance of the controller designed using feedback linearization with a simple controller based upon linearization 
about an equilibrium point, i.e., standard linear controller design. Linearizing the system about the relative equilibrium $\theta=y=0$ we obtain

$$
\left(\begin{array}{c}
\dot{\theta} \\
\ddot{\theta} \\
\dot{y}
\end{array}\right)=\left(\begin{array}{ccc}
0 & 1 & 0 \\
0 & -\frac{m_{c} v}{2 m_{c} l}+\frac{m_{w} r^{2}}{l} & -\frac{k}{\frac{l m_{c}}{3}+\frac{m_{w} r^{2}}{4 l}} \\
v & l & 0
\end{array}\right)\left(\begin{array}{c}
\theta \\
\dot{\theta} \\
y
\end{array}\right)+\left(\begin{array}{c}
0 \\
\frac{1}{\frac{l^{2} m_{c}}{3}+\frac{m_{w} r^{2}}{4}} \\
0
\end{array}\right) u .
$$

Using the standard pole placement technique, with poles at $-2,-1.414 \pm$ $1.414 i$, and $m_{c}=1.5 \mathrm{k} g, m_{w}=2.75 \mathrm{~kg}, l=0.2 \mathrm{~m}, r=0.1 \mathrm{~m}, v=1.0 \mathrm{~m} / \mathrm{s}$ and $k=75.0 \mathrm{~N} / m$, we obtain the control input

$$
u=-0.156 \theta-0.025 \dot{\theta}+1.59 y .
$$

While this controller, as expected, can stabilize the rolling motion for small deviations from the relative equilibrium, it fails to do so sufficiently far from the equilibrium. Figures 18 and 19 show the results for a system with the same physical parameters and initial conditions as the simulation in Figure 12, but where the linearized controller is used instead of the feedback linearizing controller.

\section{STABILIZATION ABOUT A NON-ZERO EQUI- LIBRIUM POINT}

In this section we present simulation results which are intended to model an attempt to stabilize the system about a point other than the equilibrium point of the non-controlled system. Such a situation would occur, for example, when a real rolling system makes a turn from a state in which it has forward velocity. In order to retain the simplicity of the model, however, we do not modify the model to directly model a turn. However, we recognize that stabilizing the system about a non-zero equilibrium point roughly corresponds to such a physical turning state because, in both cases if the system is stabilized, there will be a non-zero lateral constraint force acting on the wheel.

Note that when the system is turning (in the sense described above) the desired equiliburim point is closer to the rolling/slipping boundary than when operating in the equiliburim state $\theta=0$ and $y=0$. This is because, in contrast to the $\theta=0$ and $y=0$ equilibrium state, there will be nonzero constraint forces acting on the wheel. Thus, the turning problem is distinct because the controller may have to switch on and off many more times, and, if the desired equilibrium point is outside the rolling regime, may 
"chatter" on and off while maintaining the maximum possible deflection of the $y$ variable.

In the first simulation, the physical parameters $m_{c}=1.5 \mathrm{~kg}, m_{w}=$ $2.75 \mathrm{~kg}, l=0.2 \mathrm{~m}, r=0.1 \mathrm{~m}, k=75.0 \mathrm{~N} / \mathrm{m}, \mu_{s}=0.2, \mu_{d}=0.1$ and $v=1.0 \mathrm{~m} / \mathrm{s}$. The desired non-equilibrium point is $(\theta, y)=(0,0.25)$. If the coefficient of friction is reduced to $\mu_{s}=0.1$ and $\mu_{d}=0.05$, then the desried equilibrium point is outside the rolling regime. Figure 21 shows the chattering controller (the solid regions are the controller quickly switching between zero and the value for the rolling system). Note that the controller does not achieve the desired $y=0.25$ since it is outside the rolling regime, but maintains the system at the rolling/slipping margin.

\section{CONCLUSIONS}

In this paper, we have presented and illustrated a particular controller design for the classical shimmying wheel. The controller is guaranteed to be globally stabilizing for the wheel when it rolls without slipping, but sometimes fails to stabilize the system when the wheel is allowed to slip. However, based upon numerical simulations, the alternative control strategy seems effective in stabilizing the slipping system. We also demonstrated the efficacy of the controller in stabilizing the system about nonequilibrium operating points.

One lesson of the investigation in this paper is that for controlled rolling systems the assumption that the system is purely nonholonomic is a dangerous one. Indeed, we have demonstrated that a globally stabilizing controller for a nonholonomic system can be destabilizing for the more realistic skidding system. Thus, in cases where friction imposes the nonholonomic constraint, claims of controlled stability for such systems are only valid to the extent that the assumption regarding the nonholonomic constraint is accurate.

Of course, there are many more avenues available for further investigation. Obviously, more work is required to determine the nature of the attractor and its relationship to the purely rolling regimes where the controller is guaranteed to stabilize the system. Another avenue of study would be to study the efficacy of the controller designed here on a more realistic model of an elastic tire. Also, investigating the geometric nature of a more realistic tire model, such as presented in (Barta and Stépán, 1995) may yield insights into controller design for practical implementation. Finally, in this paper we assumed that the system transitioned from skidding to rolling when the relative velocity of the point of contact between the wheel and surface became small, which is a rather crude approximation of reality. Unfortu- 
nately, due to the inherent difficulty in modeling frictional phenomenon, a more sophisticated model would have unnecessarily complicated the model for purposes of this paper. However it would be interesting and useful, in and of itself, to completely investigate this transition.

Finally, an extremely interesting problem is whether we can prove that the alternative control strategy presented previously stabilizes the skidding system. Recall that the alternative control strategy was to allow the system to evolve in an uncontrolled manner until it switched once from skidding to rolling, at which point the controller designed for the rolling system would turned on.

If we were ablt to prove the existence of a attractor for given parameter values and also prove that the intersection of the attractor and the fourdimensional "rolling" submanifold of the phase space contains at least one point which is on or in a level surface of a Lyapunov function, such as the one presented earlier, and that the particular level set of the Lyapunov function is completely within the rolling regime, so that it would be impossible for the system to switch back to skidding, then stability for the slipping would be proven.

Other related and interesting control issues could involve attempting to directly control the attractor. This could involve controlling the rate of convergence of a solution to the attractor as well as controlling the possible chaotic behavior of the system within the attractor (which may be accomplished using the so-called OGY method (Ott et al., 1990)). Controlling the chaotic behavior within the attractor would be useful if only a small portion of the intersection of the chaotic attractor with the fourdimensional "rolling" submanifold led to stable results. Directly attempting to control the skidding system, and switching controllers between the rolling and skidding systems depending upon in which regime the system is would be another fruitful avenue of investigation. In this paper we have relied on the dissipative nature of the skidding system to drive it to the attractor wherein it will switch between rolling and skidding. Eliminating the reliance on friction and directly controlling the skidding system would likely improve the performance of the controller.

Acknowledgements: Bill Goodwine would like to thank the office of Naval Reasearch for partially supporting this work. Additionally, he would like to thank the California Institute of Technology, at which a majority of this work was performed.

Gábor Stépán would like to acknowledge the partial support of the Hungarian National Science Foundation Grant No OTKA 11524 and the GreekHungarian Science and Technology Program Grant No GR22. He would 
also like to thank the Fulbright Commission for supporting his visit at the California Institute of Technology during the first period of this research.

\section{References}

Barta, G. and Stépán, G. (1995). Untersuchung quasi-periodischer schwingungen von mit reifen versehenen radern. ZAMM, 75:S77-S78.

Bidwell, J. B. (1962). Rolling Contact Phenomena. Elsevier Publishing Company.

Blau, P. J. (1996). Friction Science and Technology. Marcel Dekker, Inc.

Böhm, F. and Kollatz, M. (1989). Some Theoretical Models for Computation of Tire Nonuniformities, volume 12.124 of Fortschrittberichte VDI. VDI Verlag.

Fliess, M., Lévine, J., Martin, P., and Rouchon, P. (1992). Sur les systèmes no linéaires différentiellement plats. C. R. Acad. Sci. Paris, 315:619624. Série I.

Gemant, A. (1950). Frictional Phenomena. Chemical Publishing, Inc.

Hauser, J., Sastry, S., and Kokotovic, P. (1992). Nonlinear control via approximate input-output linearization: The ball and beam example. IEEE Transactions on Automatic Control, 37(3):392-398.

Isidori, A. (1989). Nonlinear Control Systems. Springer-Verlag, second edition.

Nijmeijer, H. and der Schaft, A. J. V. (1990). Nonlinear Dynamical Control Systems. Springer-Verlag.

Nimark, J. I. and Fufaiv, N. A. (1972). Dynamics of Nonholonomic Systems. American Mathematical Society.

Ott, E., Grebogi, C., and Yorke, J. (1990). Controlling chaos. Physics Review Letters, 64:1196-1210.

Pacejka, H. B. (1988). Modelling of the pneumatic tyre and its impact on vehicle dynamic behaviour. Research report no. i72B, TU Delft.

Sharp, R. S. and Jones, C. J. (1980). A comparison of tyre representations in a simple wheel shimmy problem. Vehicle System Dynamics, 9:45-57. 
Stanton, T. E. (1923). Friction. Longmans, Green and Co.

Stépán, G. (1991). Chaotic motion of wheels. Vehicle System Dynamics, $20: 341-351$.

van Nieuwstadt, M., Rathinam, M., and Murray, R. M. (1998). Differential flatness and absolute equivalence. To appear, SIAM J. Control and Optimization.

van Nieuwstadt, M. J. and Murray, R. M. (1996). Real time trajectory generation for differentially flat systems. To appear, Int'l. J. Robust $\&$ Nonlinear Control. 


\section{List of Figures}

1 Wheel model. . . . . . . . . . . . . . . . . . . . . . . . . 21

2 Locally stable rolling system. . . . . . . . . . . . . . . . 22

3 Locally stable rolling system. . . . . . . . . . . . . . . . 23

4 Locally unstable rolling system. . . . . . . . . . . . . . . . 24

5 Locally unstable rolling system. . . . . . . . . . . . . . . 25

6 Unstable limit cycle. . . . . . . . . . . . . . . . . . . 26

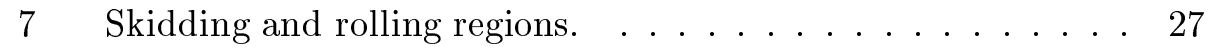

8 Skidding and rolling regions for $y=0 \ldots \ldots \ldots \ldots$

$9 \quad$ Rolling and skidding system. . . . . . . . . . . . . . . . 29

10 Rolling and skidding system. . . . . . . . . . . . . 30

11 Controlled rolling system. . . . . . . . . . . . . . 31

12 Controlled rolling system $\left(l=0.152<l_{c r}\right.$, unstable case). . . 32

13 Controlled skidding system. . . . . . . . . . . . . . 33

14 Controlled skidding system. . . . . . . . . . . . . . . . 34

15 Controlled skidding system: Alternative control strategy. . . . 35

16 Controlled skidding system: Alternative control strategy. . . . 36

17 Controlled skidding system: Alternative control strategy. . . . 37

18 Simple pole placement controller. . . . . . . . . . . . . . . . 38

19 Simple pole placement controller. . . . . . . . . . . . . . . 39

20 Controlled turning system: Feedback linearization controller. 40

21 Controlled turning system: Small coefficient of friction. . . . . 41 

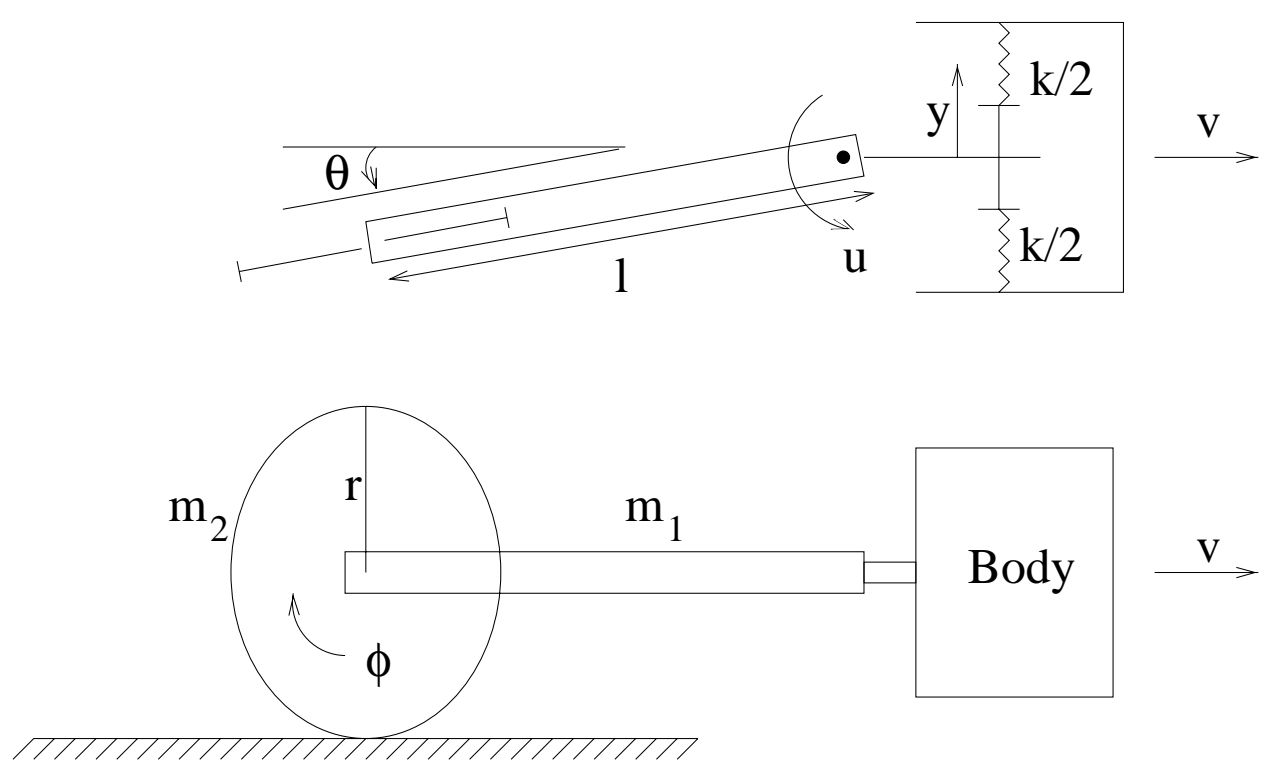

Figure 1. Wheel model. 

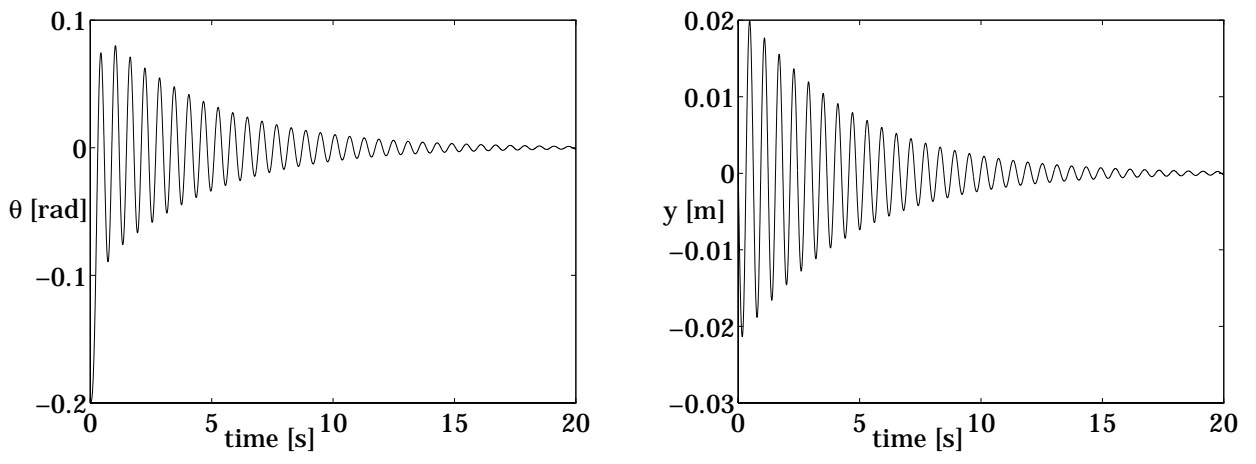

Figure 2. Locally stable rolling system. 


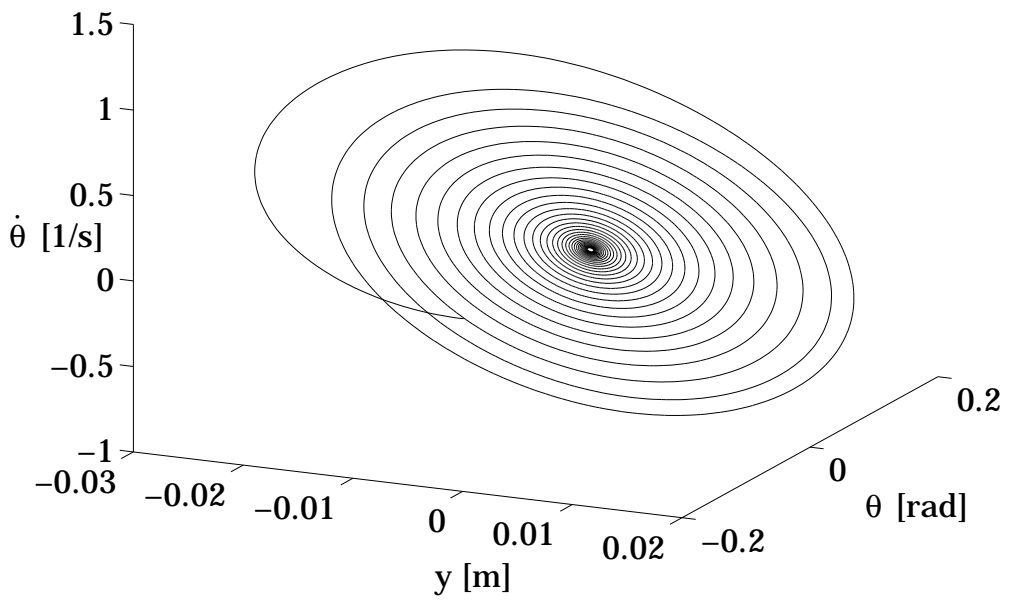

Figure 3. Locally stable rolling system. 

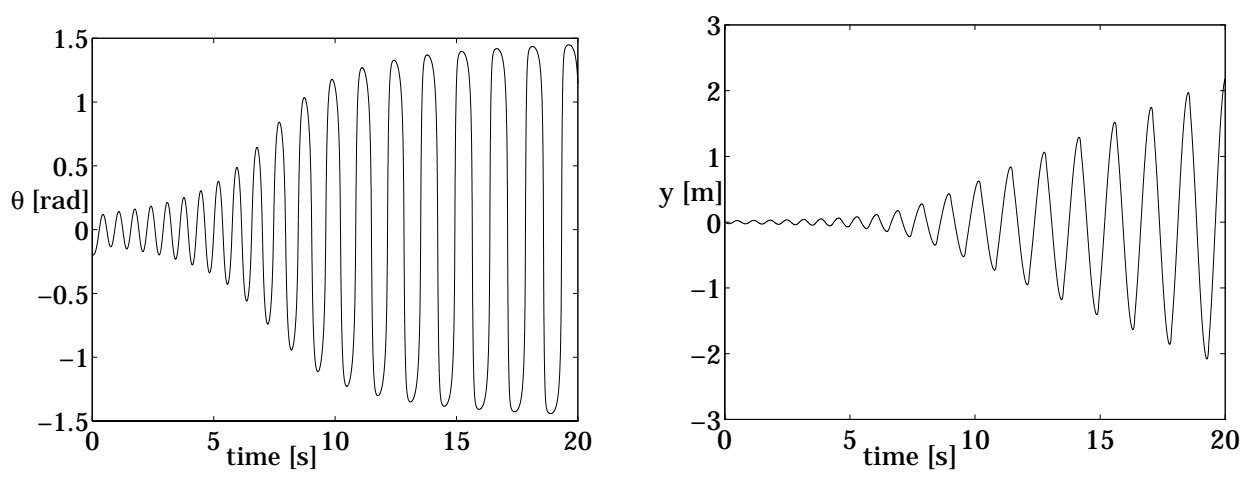

Figure 4. Locally unstable rolling system. 


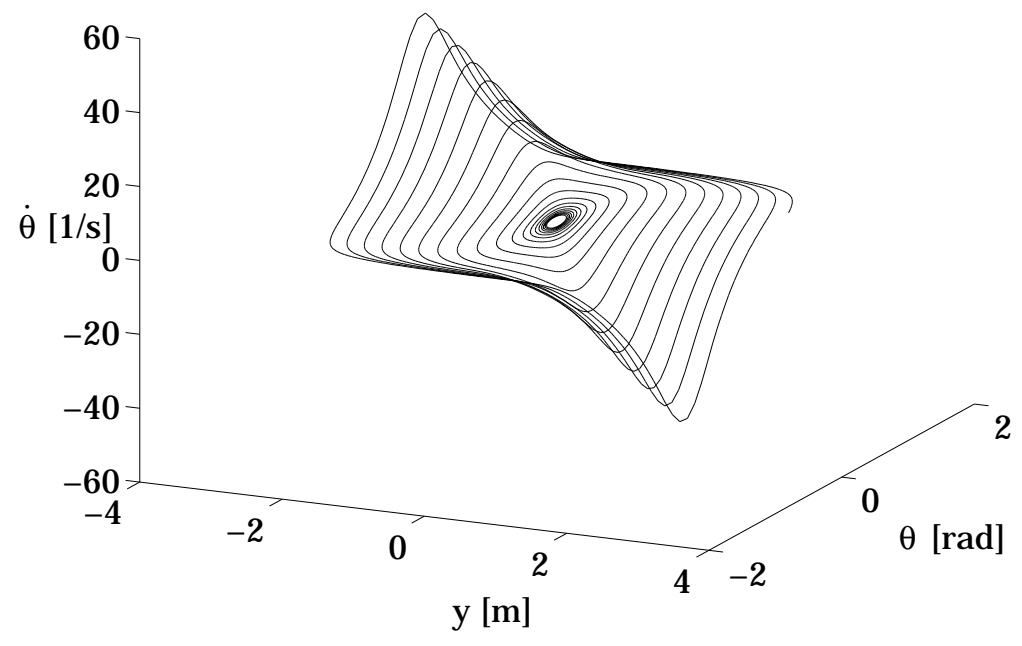

Figure 5. Locally unstable rolling system. 


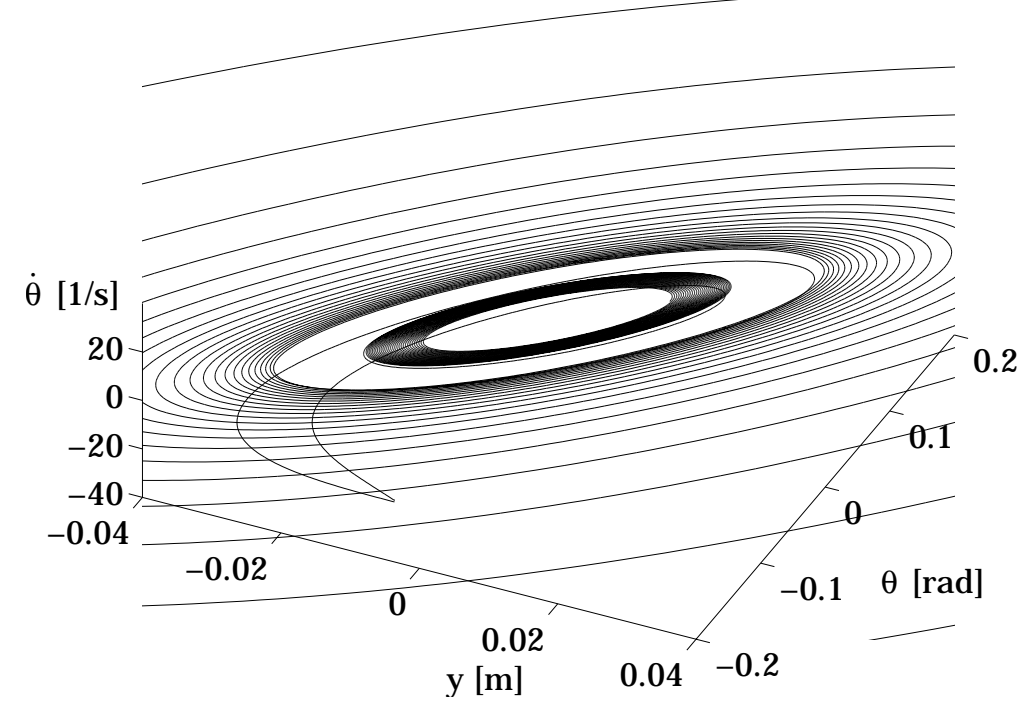

Figure 6. Unstable limit cycle. 

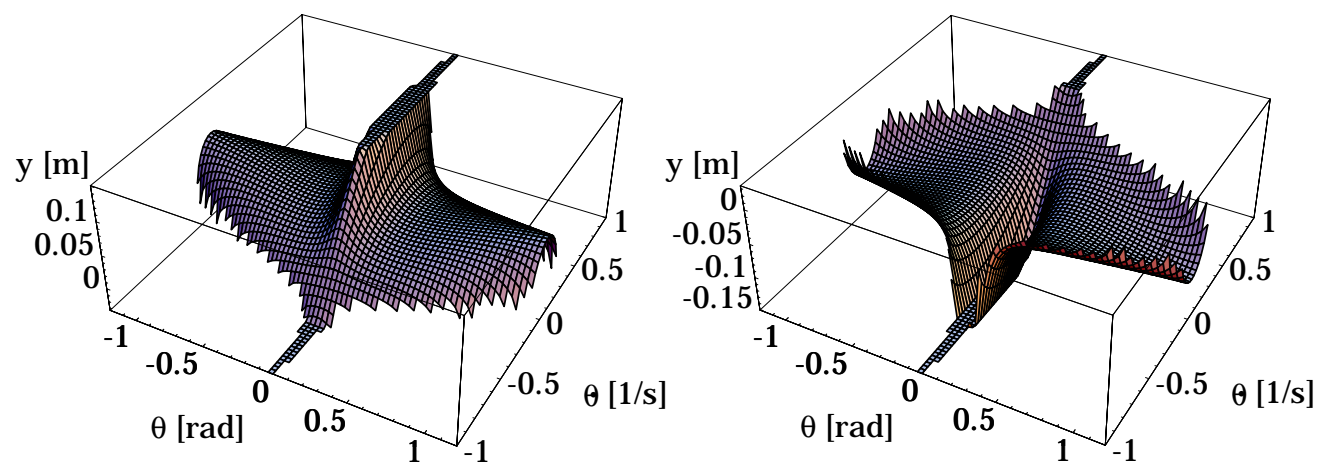

Figure 7. Skidding and rolling regions. 


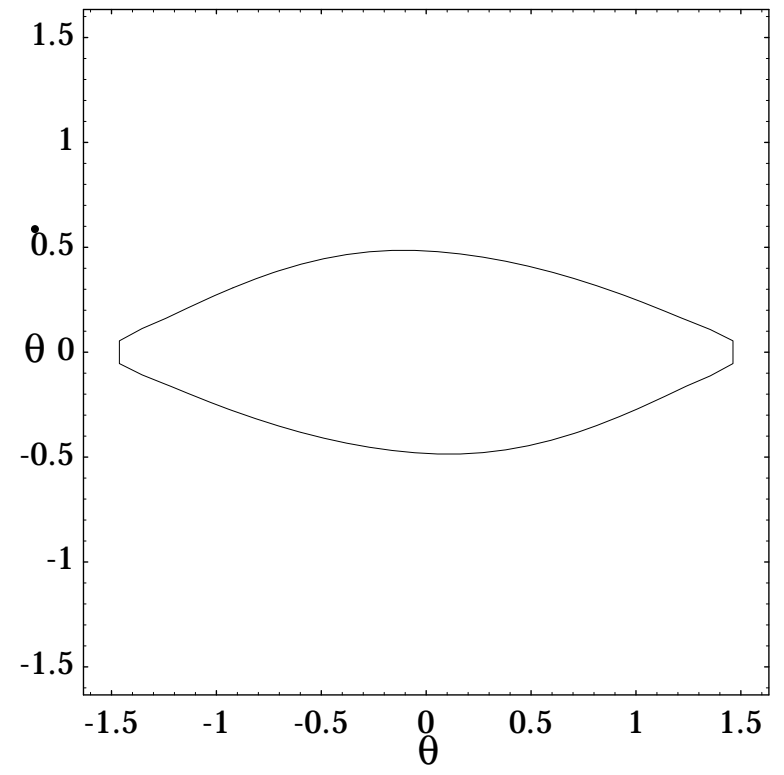

Figure 8. Skidding and rolling regions for $y=0$. 


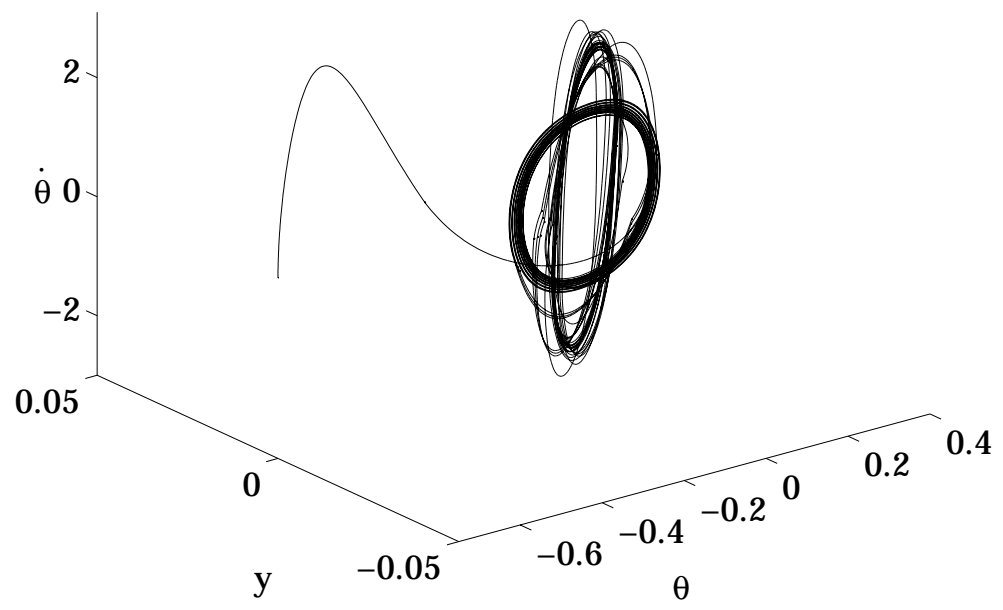

Figure 9. Rolling and skidding system. 


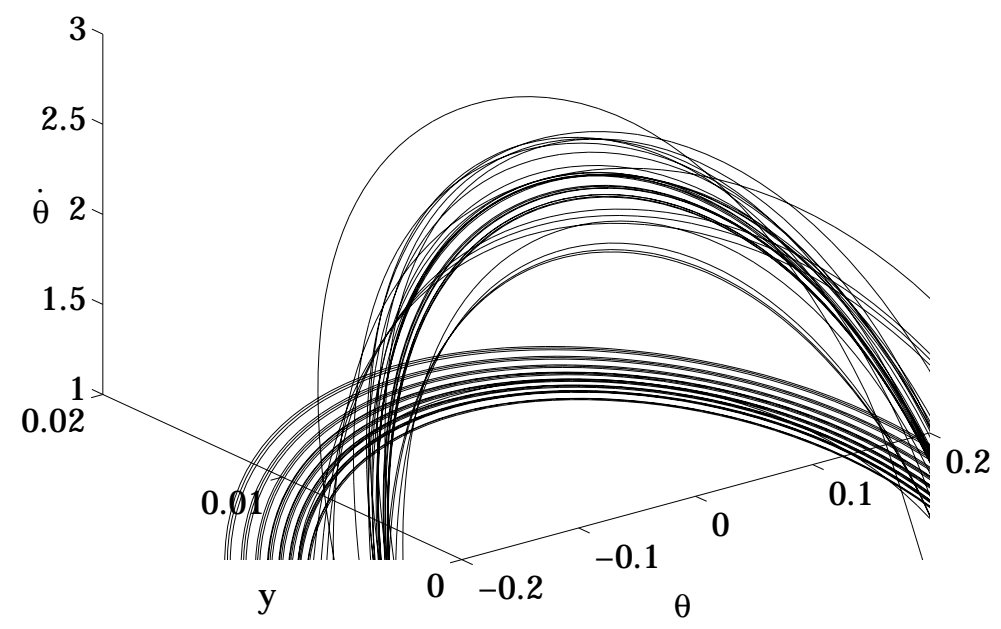

Figure 10. Rolling and skidding system. 

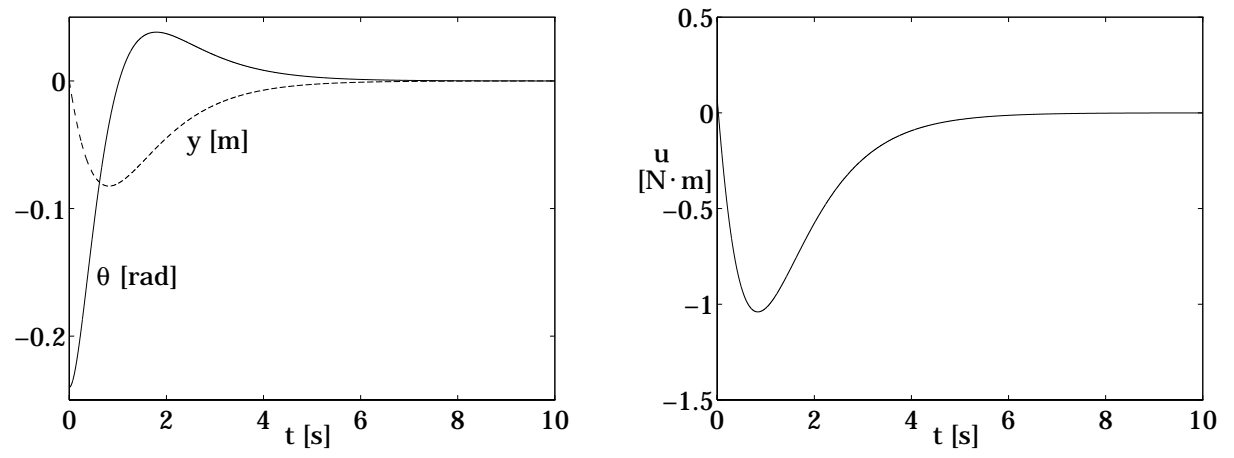

Figure 11. Controlled rolling system. 

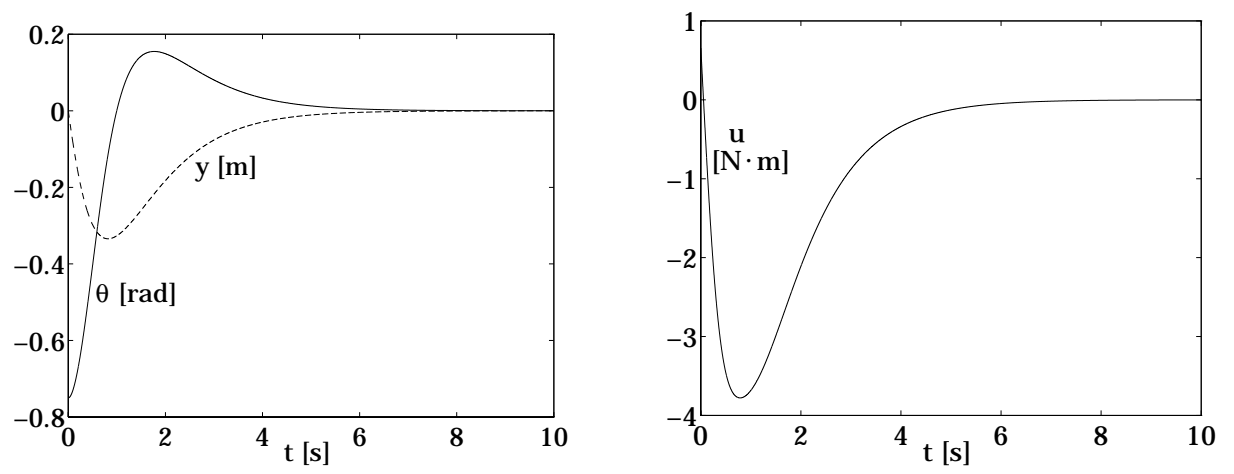

Figure 12. Controlled rolling system $\left(l=0.152<l_{c r}\right.$, unstable case). 

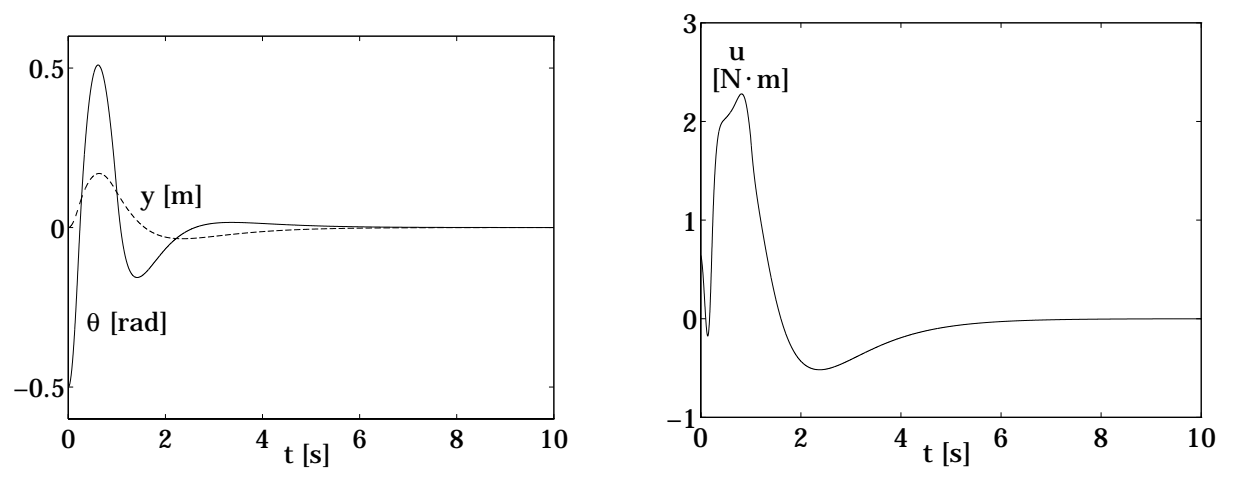

Figure 13. Controlled skidding system. 

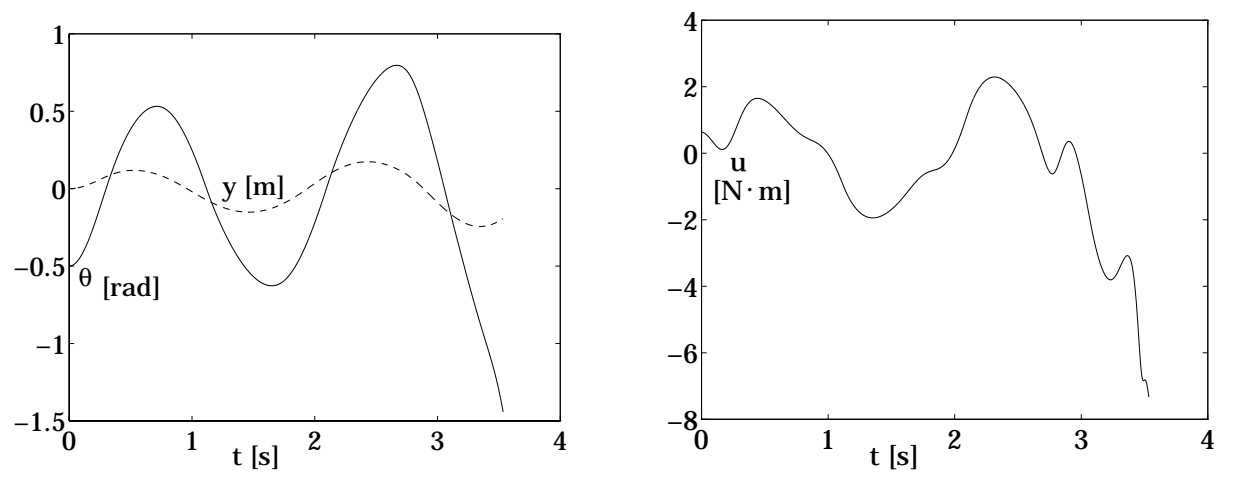

Figure 14. Controlled skidding system. 

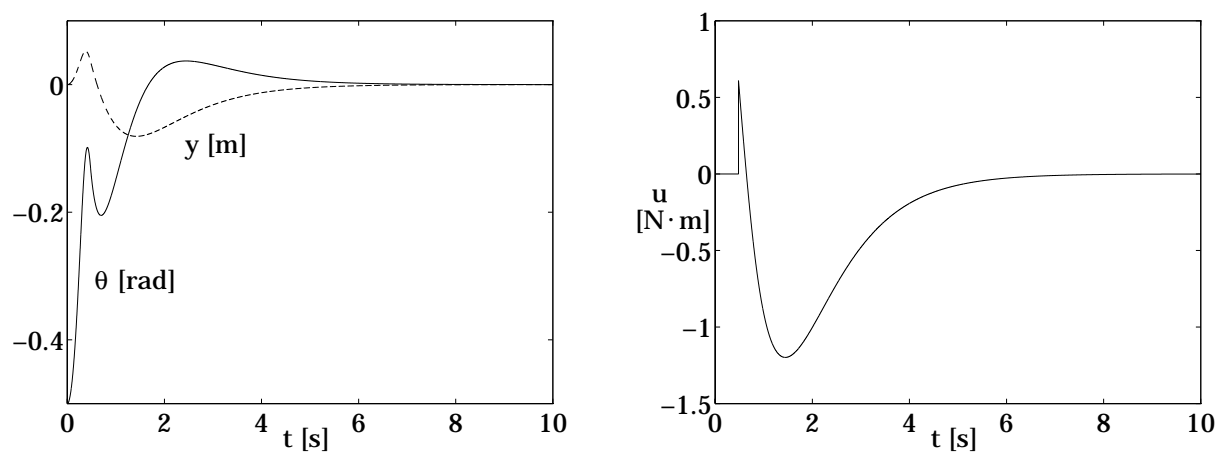

Figure 15. Controlled skidding system: Alternative control strategy. 

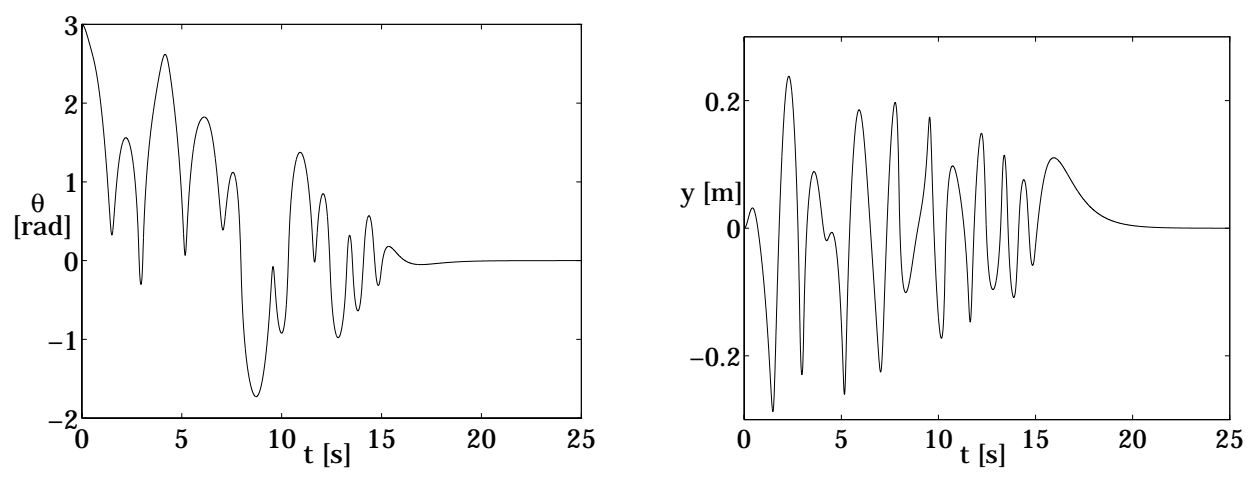

Figure 16. Controlled skidding system: Alternative control strategy. 


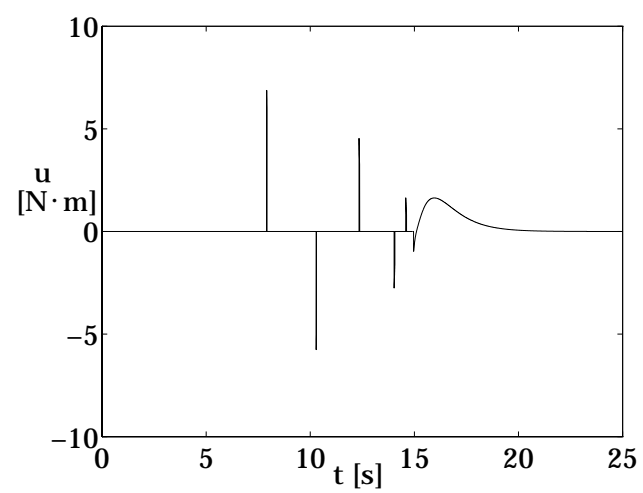

Figure 17. Controlled skidding system: Alternative control strategy. 

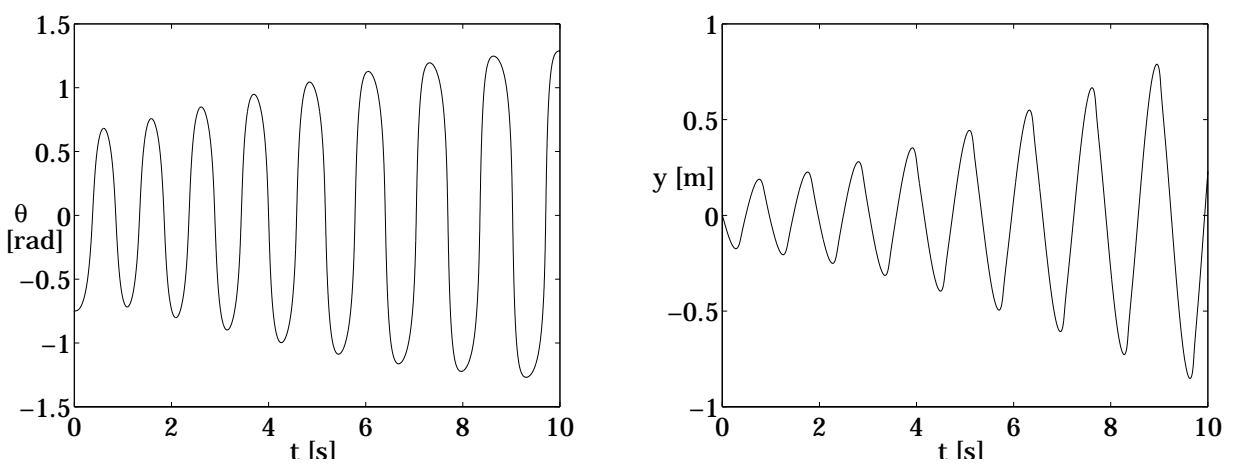

Figure 18. Simple pole placement controller. 


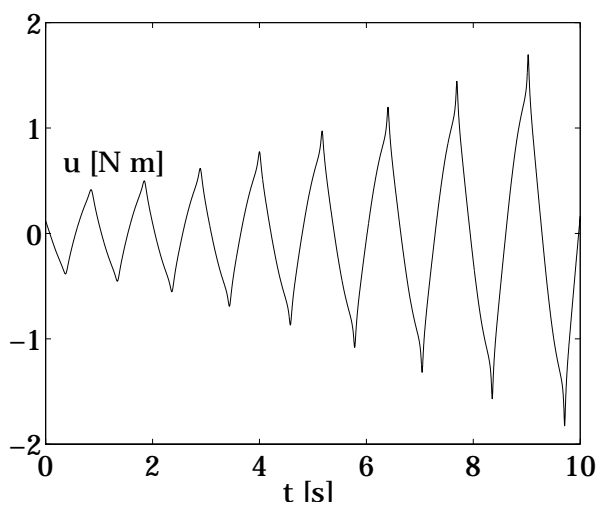

Figure 19. Simple pole placement controller. 

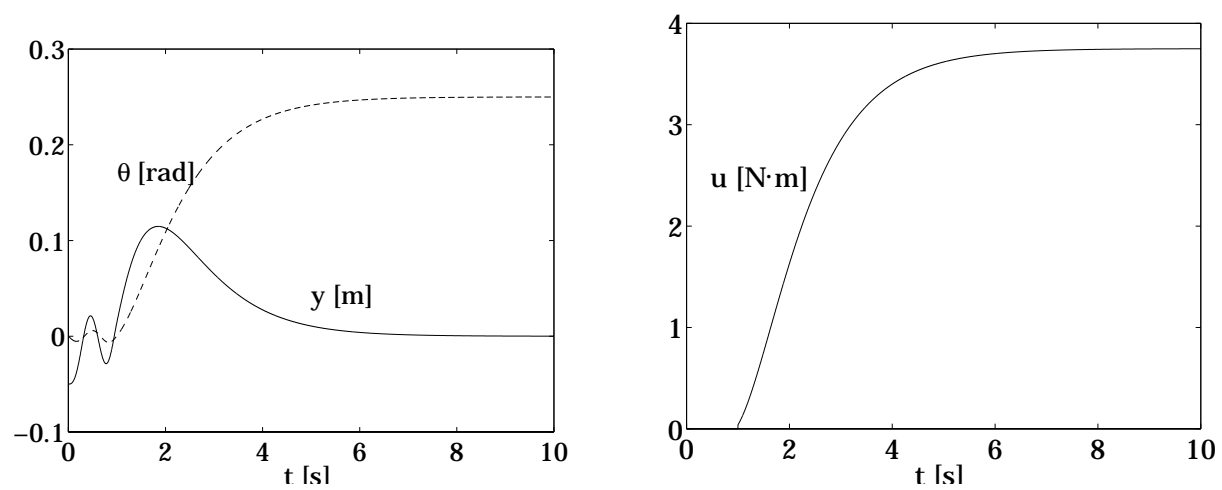

Figure 20. Controlled turning system: Feedback linearization controller. 

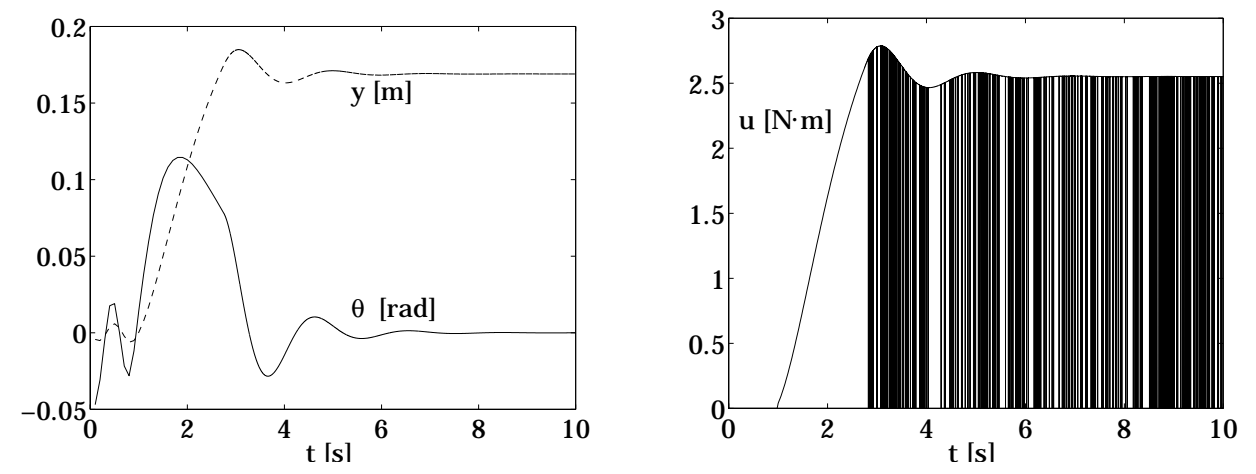

Figure 21. Controlled turning system: Small coefficient of friction. 